\title{
BOUNDS FOR THE FUNDAMENTAL SOLUTION OF A PARABOLIC EQUATION ${ }^{1}$
}

\author{
BY D. G. ARONSON
}

\author{
Communicated by J. K. Moser, June 19, 1967
}

1. Introduction. It is well known that the parabolic equation

$$
a_{i j}(x, t) u_{x_{i} x_{j}}+a_{i}(x, t) u_{x_{i}}+a(x, t) u-u_{t}=0
$$

possesses a fundamental solution provided that the coefficients are Hölder continuous. Here $x=\left(x_{1}, \cdots, x_{n}\right)$ denotes a point in $E^{n}$ with $n \geqq 1, t$ denotes a point on the real line, and we employ the convention of summation over repeated indices. The fundamental solution $g(x, t ; \xi, \tau)$ can be constructed by the classical parametrix method, and it satisfies the inequality $0 \leqq g \leqq K \gamma$, where $\gamma$ is the fundamental solution of $\alpha \Delta u=u_{t}$ for some constant $\alpha>0$ and $K>0$ is a constant which depends upon the Hölder norms of the coefficients ([4], [5]). Several authors have investigated the problem of bounding $g$ from below. Il'in, Kalashnikov, and Oleinik [5] proved that $g \geqq$ const $(t-\tau)^{-n / 2}$ in the paraboloid $|x-\xi|^{2} \leqq \operatorname{const}(t-\tau)$; while Besala [3] and Friedman [4] have derived lower bounds for $g$ which are valid when $t-\tau$ is bounded away from zero. In the appendix to his important paper [6] on Hölder continuity of solutions of parabolic and elliptic equations, Nash asserts the existence of global upper and lower bounds for the fundamental solution of the divergence structure parabolic equation

$$
u_{t}-\left\{a_{i j}(x, t) u_{x_{i}}\right\}_{x_{j}}=0 .
$$

These bounds do not involve the fundamental solutions of equations of the form $\alpha \Delta u=u_{t}$ and thus are not as sharp as the classical upper bound for (1). On the other hand, Nash's estimates are independent of the moduli of continuity of the coefficients of (2). tion

For $(x, t)$ in the strip $S=E^{n} \times(0, T)$, consider the parabolic equa-

$$
u_{t}-\left\{a_{i j}(x, t) u_{x_{i}}+a_{j}(x, t) u\right\}_{x_{j}}-b_{j}(x, t) u_{x_{j}}-c(x, t) u=0 .
$$

Assume that the coefficients of (3) are bounded measurable functions of $(x, t)$ in $S$ and that there exists a constant $\nu>0$ such that $a_{i j}(x, t) \zeta_{i} \zeta_{j}$ $\geqq \nu^{-1}|\zeta|^{2}$ almost everywhere in $S$ for all $\zeta \in E^{n}$. Let $g(x, t ; \xi, \tau)$ denote.

1 This research was partially supported by the United States Air Force Office of Scientific Research under Contract No. AF-AFOSR 883-65. 
the fundamental solution of (3). If the coefficients of (3) are smooth, then $g$ exists in the classical sense, otherwise it must be interpreted in the weak sense (cf. [1]). The purpose of this note is to announce the following global bounds for $g$.

THEOREM 1. There exist positive constants $\alpha_{1}, \alpha_{2}$, and $K$ such that

$$
K^{-1} \gamma_{1}(x-\xi, t-\tau) \leqq g(x, t ; \xi, \tau) \leqq K \gamma_{2}(x-\xi, t-\tau)
$$

for all $(x, t),(\xi, \tau) \in S$ with $t>\tau$, where $\gamma_{i}(x, t)$ is the fundamental solution of $\alpha_{i} \Delta u=u_{t}$ for $i=1,2$. The constants depend only on $\nu, n, T$ and the bounds for the coefficients of (3).

An analogous result also holds for the Green's function of (3) in a cylindrical domain $\Omega \times(0, T)$ provided that $x$ and $\xi$ are at a positive distance $\delta$ from $\partial \Omega$. In this case the constants will also depend upon $\delta$. Moreover, it is not necessary that the coefficients $a_{j}, b_{j}$ and $c$ be bounded. It suffices that they belong to certain Lebesgue classes and satisfy an algebraic condition. The proofs of Theorem 1 and its extensions will be published in detail elsewhere. Here we shall prove Theorem 1 for the special case of equation (2). In this special case we encounter the main ideas of the general proof without too much technical detail. In both the general case and the special case we make essential use of several results proved in reference [2].

To avoid the complication of having to consider the weak fundamental solution of (2), we make the qualitative assumption that the coefficients of (2) are smooth, say, $a_{i j} \in C^{\infty}(S)$. Moreover, to simplify the computations we assume $a_{i j}=a_{j i}$ in $S$. The quantitative assumptions can thus be put in the following form. There exists a constant $\nu \geqq 1$ such that

$$
\nu^{-1}|\zeta|^{2} \leqq a_{i j}(x, t) \zeta_{i} \zeta_{j} \leqq \nu|\zeta|^{2} \quad \text { for all }(x, t) \in S \text { and } \zeta \in E^{n}
$$

Under these conditions the fundamental solution $g(x, t ; \xi, \tau)$ exists, and it is known that

$$
\int_{E^{n}} g(x, t ; \xi, \tau) d \xi=\int_{E^{n}} g(x, t ; \xi, \tau) d x=1 .
$$

In addition, Nash [6] has shown that

$$
\int_{E^{n}} g^{2}(x, t ; \xi, \tau) d \xi \leqq k(t-\tau)^{-n / 2}, \quad \int_{E^{n}} g^{2}(x, t ; \xi, \tau) d x \leqq k(t-\tau)^{-n / 2}
$$

and 


$$
g(x, t ; \xi, \tau) \leqq k(t-\tau)^{-n / 2},
$$

where $k$ denotes a positive constant which depends only upon $n$ and $\nu$.

2. The lower bound. According to Theorem $7^{\prime}$ of [2], if

$$
\mathscr{T C}=\inf _{0<t<T} \int_{|x-\xi|^{2}<\alpha(t-\tau)} g(x, t ; \xi, \tau) d x>0
$$

for some $\alpha>0$, then there exist constants $C_{1}$ and $C_{2}$ such that

$$
g(x, t ; \xi, \tau) \geqq C_{1}(t-\tau)^{-n / 2} \exp \left\{-C_{2}|x-\xi|^{2} /(t-\tau)\right\}
$$

for $(x, t),(\xi, \tau) \in S$ with $t>\tau$. Here $C_{1}$ depends only on $\alpha, \mathfrak{T}, n$, and $\nu$, while $C_{2}$ depends only on $n$ and $\nu$. Hence, in order to prove the validity of the lower bound in Theorem 1 it suffices to show that (8) holds.

Let $(\xi, \tau) \in S$ and $t \in(\tau, T)$ be fixed. Consider the function

$$
v(y, s)=\int_{|x-\xi|^{2}<\alpha(t-\tau)} g(x, t ; y, s) d x
$$

for $s<t$, where $\alpha=16 / T$. Note that $v$ is a solution of the equation $v_{s}+\left(a_{i j} v_{y_{i}}\right)_{y_{j}}=0$ adjoint to $(2)$ for $(y, s) \in E^{n} \times[0, t)$ with initial values

$$
\begin{aligned}
v(y, t) & =1 \text { if }|y-\xi|^{2}<\alpha(t-\tau), \\
& =0 \text { if }|y-\xi|^{2}>\alpha(t-\tau) .
\end{aligned}
$$

Set

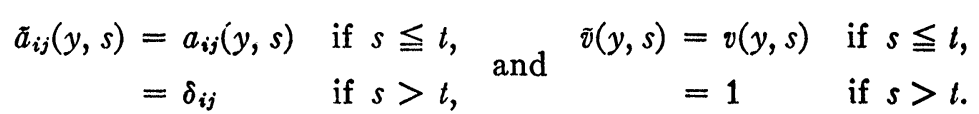

Then $v$ is a nonnegative weak solution (in the sense of [2]) of the equation

$$
\tilde{v}_{s}+\left\{\tilde{a}_{i j}(y, s) \tilde{v}_{y_{i}}\right\}_{y j}=0
$$

in the cylinder $\left\{|y-\xi|^{2}<\alpha(t-\tau)\right\} \times[0, \infty)$. We now apply the Harnack inequality for weak solutions of (9). By Theorem 5 of [2] with $\delta^{2}=R=\alpha(t-\tau) / 16 \leqq \alpha T / 16=1$ we have

$$
\int_{|x-\xi|^{2}<\alpha(t-\tau)} g(x, t ; \xi, \tau) d x=\tilde{v}(\xi, \tau) \geqq \tilde{v}(\xi, t) e^{-C(16 / \alpha+1)}=e^{-C(T+1)},
$$

where, for equations of the form (9), $C$ depends only upon $n$ and $\nu$. 
3. An auxiliary estimate. To obtain the upper bound for $g$ we shall use the following estimate for a solution of the Cauchy problem for (2) with data whose support lies outside a sphere in $E^{n}$. The theorem which we give here is a special case of a more general result which is valid for solutions of quasilinear equations of the type considered in [2].

THEOREM 2. Let $u_{0}(x)$ be an $L^{2}\left(E^{n}\right)$ function such that $u_{0}=0$ for $|x-y|<\sigma$, where $y \in E^{n}$ and $\sigma>0$ are fixed. Suppose that $u$ is a solution of (2) in $E^{n} \times(\eta, T)$ with initial values $u(x, \eta)=u_{0}(x)$. Then if $u \in L^{\infty}\left(E^{n} \times(\eta, s)\right)$ for any $s$ which satisfies $0<s-\eta \leqq \sigma^{2}$, we have

$$
|u(y, s)| \leqq k(s-\eta)^{-n / 4} \exp \left[-\sigma^{2} / 32 \nu(s-\eta)\right]\left\|u_{0}\right\|_{L^{2}\left(E^{n}\right)}
$$

where $k>0$ is a constant which depends only upon $n$ and $\nu$.

Proof. For $R \geqq 2$, let $\gamma_{R}(x)$ be a $C^{\infty}\left(E^{n}\right)$ function such that $\gamma_{R} \equiv 1$ for $|x-y| \leqq R-1, \gamma_{R} \equiv 0$ for $|x-y| \geqq R, 0 \leqq \gamma_{R} \leqq 1$ and $\left|\operatorname{grad} \gamma_{R}\right|$ is bounded by a constant independent of $R$. Multiply both sides of (2) by $\gamma_{R}^{2} e^{2 h} u$, where

$$
h(x, t)=-|x-y|^{2} / 8 v\{2(s-\eta)-(t-\eta)\},
$$

and integrate over $E^{n} \times(\eta, \tau)$. After integration by parts and some elementary estimates, we obtain

$$
\begin{aligned}
& \left.\frac{1}{2} \int \gamma_{R}^{2} e^{2 h} u^{2}\right|_{t=\tau} d x-\iint \gamma_{R}^{2} e^{2 h} u^{2}\left(2 a_{i j} h_{x_{i}} h_{x_{j}}+h_{t}\right) d x d t \\
& \quad \leqq\left.\frac{1}{2} \int \gamma_{R}^{2} e^{2 h} u^{2}\right|_{t \rightarrow n} d x+2 \nu \iint_{E^{n} \times(\eta, s)} e^{2 h} u^{2}\left|\operatorname{grad} \gamma_{R}\right|^{2} d x d t .
\end{aligned}
$$

In view of (4), $2 a_{i j} h_{x_{i}} h_{x_{j}}+h_{t} \leqq 0$. Moreover, since $u \in L^{\infty}\left(E^{n} \times(\eta, s)\right)$, the second integral on the right in (10) tends to zero as $R \rightarrow \infty$. Therefore we derive from (10) the estimate

$$
\begin{aligned}
\max _{(\eta, 8)} \int_{|x-y|^{2} \leq(8-\eta) / 4} & \exp [2 h(x, t)] u^{2}(x, t) d x \\
& \leqq \int \exp [2 h(x, \eta)] u_{0}^{2}(x) d x=\int_{|x-y| z o} \exp [2 h(x, \eta)] u_{0}^{2}(x) d x .
\end{aligned}
$$

For $|x-y|^{2} \leqq(s-\eta) / 4$ and $\eta \leqq t \leqq s$ it is easily seen that $2 h(x, t)$ $\geqq-1 / 16 \nu$, while for $|x-y|^{2} \geqq \sigma^{2} \geqq s-\eta$ we have $2 h(x, \eta) \leqq-1 / 16 \nu$ $-\sigma^{2} / 16 \nu(s-\eta)$. Thus 
(11) $\max _{(\eta, s)} \int_{|x-\nu|^{2} \leqq(s-\eta) / 4} u^{2}(x, t) d x \leqq \exp \left[-\sigma^{2} / 16 \nu(s-\eta)\right]\left\|u_{0}\right\|^{2} L^{2}\left(E^{n}\right)$.

Let $\left.Q=\left\{|x-y|^{2}<(s-\eta) / 4\right\} \times(\eta, s)\right]$. According to Theorem 2 of $[2]$,

$$
|u(y, s)| \leqq C(s-\eta)^{-(n+2) / 4}\left(\iint_{Q} u^{2} d x d t\right)^{1 / 2}
$$

where $C$ depends only upon $n$ and $\nu$. In view of (11)

$$
\left(\iint_{Q} u^{2} d x d t\right)^{1 / 2} \leqq(s-\eta)^{1 / 2} \exp \left[-\sigma^{2} / 32 \nu(s-\eta)\right]\left\|u_{0}\right\|_{L^{2}\left(E^{n}\right)}
$$

and the assertion of the theorem follows by combining the last two estimates.

It is clear that a result analogous to Theorem 2 also holds for the equation adjoint to (2).

4. The upper bound. We first prove that if $\sigma^{2} \geqq s-\eta>0$ then

$$
\int_{|y-\zeta|>\sigma} g^{2}(y, s ; \zeta, \eta) d \zeta \leqq k(s-\eta)^{-n / 2} \exp \left[-\sigma^{2} / 16 \nu(s-\eta)\right],
$$

where $k>0$ is a constant depending only upon $n$ and $\nu$. To this end set

$$
u(x, t)=\int_{|y-\zeta|>\sigma} g(x, t ; \zeta, \Re) g(y, s ; \zeta, \Re) d \zeta .
$$

Then $u$ is a nonnegative solution of (2) for $t>\eta$ with initial values $u(x, \eta)=0$ if $|x-y|<\sigma$, and $u(x, \eta)=g(y, s ; x, \eta)$ if $|x-y|>\sigma$. Moreover, in view of (5), (6), and (7), $u(x, \eta) \in L^{2}\left(E^{n}\right)$ and $0 \leqq u(x, t)$ $\leqq k(s-\eta)^{-n / 2}$. Thus we may apply Theorem 2 to obtain

$$
\begin{aligned}
0 \leqq u(y, s) & =\int_{|y-\zeta|>\sigma} g^{2}(y, s ; \zeta, \eta) d \zeta \\
& \leqq k(s-\eta)^{-n / 4} \exp \left[-\sigma^{2} / 32 \nu(s-\eta)\right]\left\{\int_{|y-\zeta|>\sigma} g^{2}(y, s ; \zeta, \eta) d \zeta\right\}^{1 / 2}
\end{aligned}
$$

and (12) follows easily. Note that a similar estimate holds if we integrate with respect to $y$ instead of $\zeta$ in (12).

Let $(x, t),(\xi, \tau)$ be fixed points of $S$ with $t>\tau$. Set $\sigma=|x-\xi| / 2$ and assume that $t-\tau \leqq \sigma^{2}$. By the Kolmogorov identity

$$
g(x, t ; \xi, \tau)=\int_{E^{n}} g(x, t ; \zeta,(t+\tau) / 2) g(\zeta,(t+\tau) / 2 ; \xi, \tau) d \zeta
$$


Split the integral over $E^{n}$ into an integral $J_{1}$ over $|x-\zeta| \geqq \sigma$ and an integral $J_{2}$ over $|x-\zeta|<\sigma$. By the Schwarz inequality

$$
\begin{aligned}
& J_{1} \leqq\left\{\int_{|x-\zeta| \geq \sigma} g^{2}(x, t ; \zeta,(t+\tau) / 2) d \zeta\right\}^{1 / 2} \\
& \cdot\left\{\int_{|x-\zeta| \geq \sigma} g^{2}(\zeta,(t+\tau) / 2 ; \xi, \tau) d \zeta\right\}^{1 / 2} .
\end{aligned}
$$

Now using (12) to estimate the first integral on the right and (6) to estimate the second, we obtain

$$
J_{1} \leqq k(t-\tau)^{-n / 2} \exp \left\{-|x-\xi|^{2} / 64 \nu(t-\tau)\right\},
$$

where $k$ depends only upon $n$ and $\nu$. The estimate (13) also holds for $J_{2}$. To show this we note that $|x-\zeta|<\sigma=|x-\xi| / 2$ implies that $|\xi-\zeta| \geqq \sigma$. Thus $J_{2}$ is dominated by the integral over $|\xi-\zeta| \geqq \sigma$. The assertion now follows by the argument used above with the roles of (12) and (6) interchanged. Thus we have derived the required upper bound for $g$ in case $|x-\xi|^{2} \geqq 4(t-\tau)$. If $|x-\xi|^{2}<4(t-\tau)$, then in view of (7), we have

$g(x, t ; \xi, \tau) \leqq k(t-\tau)^{-n / 2} \leqq k e^{-1 / 16 v}(t-\tau)^{-n / 2} \exp \left\{-|x-\xi|^{2} / 64 \nu(t-\tau)\right\}$,

where $k$ depends only upon $n$ and $\nu$. This completes the proof of Theorem 1 for the special case of equation (2).

5. Remark. In the estimates derived above all of the constants are independent of $T$ except for the lower bound for

$$
\int_{|x-\xi|^{2}<\alpha(t-\tau)} g(x, t ; \xi, \tau) d x
$$

in §2. It is, however, possible to choose $\alpha>0$ depending only upon $n$ and $\nu$ so that the lower bound for this integral also depends only upon $n$ and $\nu$. Thus all of the estimates can be made independent of $T$.

Consider the equation

$$
u_{t}-\left\{a_{i j}(x) u_{x_{i}}\right\}_{x_{j}}=0
$$

in $E^{n} \times(0, \infty)$, where the $a_{i j}$ satisfy (4) for all $x \in E^{n}$ and $n \geqq 3$. Then

$$
\int_{0}^{\infty} g(x, t ; \xi, 0) d t=G(x, \xi),
$$

where $G(x, \xi)$ is the fundamental solution of the elliptic equation

$$
\left\{a_{i j}(x) u_{x_{i}}\right\}_{x_{s}}=0 \text {. }
$$


Since the constants in the estimates for $g$ are independent of $T$ we can integrate these estimates to obtain

$$
K^{-1}|x-\xi|^{2-n} \leqq G(x, \xi) \leqq K|x-\xi|^{2-n} .
$$

where $K$ depends only on $n$ and $\nu$. This result is known, having been derived directly from potential theoretic considerations by $\mathrm{H}$. Royden and by Littman, Stampacchia and Weinberger.

\section{REFERENCES}

1. D. G. Aronson, Isolated singularities of solutions of second order parabolic equations, Arch. Rational Mech. Anal. 19 (1965), 231-238.

2. D. G. Aronson and James Serrin, Local behavior of solutions of quasilinear parabolic equations, Arch. Rational Mech. Anal. 25 (1967), 81-122.

3. P. Besala, On a certain property of the fundamental solution of a linear parabolic equation the last coefficient of which is unbounded, Bull. Acad. Polon. Sci. Sér. Sci. Math. Astronom. Phys. 11 (1963), 155-158.

4. A. M. Il'in, A.S. Kalashnikov and O. A. Oleinik, Second order linear equations of parabolic type, Russian Math. Surveys 17 No. 3 (1962), 1-143.

5. Avner Friedman, Partial differential equations of parabolic type, Prentice-Hall, Englewood Cliffs, N. J., 1964.

6. J. Nash, Continuity of solutions of parabolic and elliptic equations, Amer. J. Math. 80 (1958), 931-954.

UNIVERSITY OF MiNNESOTA 